\title{
Uncertainty and universality in the power-law singularity as a precursor of catastrophic rupture
}

\author{
JIN Yuan ${ }^{1,2}$, XIA MengFen $^{1} \&$ WANG HaiYing ${ }^{1 *}$ \\ ${ }^{1}$ State Key Laboratory of Nonlinear Mechanics, Institute of Mechanics, Chinese Academy of Sciences, Beijing 100190, China, \\ ${ }^{2}$ Graduate University of Chinese Academy of Sciences, Beijing 100049, China
}

Received January 16, 2012; accepted March 9, 2012; published online April 20, 2012

\begin{abstract}
The prediction of catastrophic rupture of heterogeneous brittle material has been investigated by researchers in the past. In this work, the acoustic emission generated by marble samples under compression was analyzed to verify a power law singularity of index $-1 / 2$ as a catastrophe precursor. It is found that prior to catastrophe, the variation of the system response to the controlling parameter follows a power law of negative index, which proves the power law singularity as a common precursor of catastrophe. However, the power indexes vary with variables and samples. The uncertainty reflects sample specificity of an evolution induced catastrophe (EIC) process.
\end{abstract}

uncertainty, universality, power-law singularity, catastrophic rupture, acoustic emission

PACS number(s): 62.20MK, 46.65+g, 43.40.Le

Citation: Jin Y, Xia M F, Wang H Y. Uncertainty and universality in the power-law singularity as a precursor of catastrophic rupture. Sci China-Phys Mech Astron, 2012, 55: 1098-1102, doi: 10.1007/s11433-012-4727-4

The prediction of catastrophic rupture in heterogeneous brittle material such as large earthquake events has eluded generations of seismologists, physicists, and mechanical engineers [1-3]. However, the fundamental difficulties are evident. Heterogeneous brittle material, like rock, has intrinsically complex structure and numerous microdamages at the mesoscopic scale. The catastrophic failure actually results from a cascade evolution of microdamages from small to large scales in the case far from equilibrium, which magnifies the effects of microstructures on failure and induces trans-scale sensitivity [4-7]. Hence it is imperative that we develop new models or methods to deal with the trans-scaled sensitivity.

Motivated by the above-mentioned facts, researchers have proposed various statistical models to study the catastrophic failure of brittle material. Some models character-

*Corresponding author (email: why@1nm.imech.ac.cn) ize the collective effects of microdamage with the formulation of statistical microdamage mechanics [8,9]. Some models compare the fracture to either the critical point at a phase transition or the percolation phenomenon. Others have suggested the attractor in a self-organized critical system $[10,11]$. In addition, experimental observations prove that there are some precursors prior to a catastrophic rupture or major earthquake, such as accelerating moment release or power law increase in the number of intermediate-size events, anomalously high values of load/unload response ratio (LURR) and critical sensitivity [12-17].

Based on statistical microdamage mechanics, Rong [18] suggested that the power law singularity with index $-1 / 2$ be an effective precursor before catastrophic rupture. To verify the universality of power law singularity, in this paper, the damage evolution during the catastrophic rupture of marble samples under uniaxial compression is analyzed. The results prove power law singularity as a universal precursory feature of catastrophe, but with uncertainty in the power index. 


\section{Precursor of power law singularity}

The catastrophic rupture of a brittle material stems from random spatially distributed microdamage evolution including nucleation, growth and coalescence. If the discrete nature of the microdamage evolution process is negligible, the collective effects of microdamage evolution could be described with a continuous model of statistical microdamage mechanics. Hence, in the case of quasi-static and monotonic loading, the accumulative response of the system $Z$ can be denoted with a continuous function of the controlling parameter $\lambda(\mathrm{d} \lambda / \mathrm{d} t>0)$ as $Z=Z(\lambda)$, or inversely, $\lambda=\lambda(Z)$. Here, $Z$ can be the accumulative damage, strain, energy release of the loading system. Assuming that the controlling parameter and accumulative response of the system at the catastrophic rupture be $\lambda_{F}$ and $Z_{F}$, respectively, we can expand the function in the vicinity of the catastrophic rupture,

$$
\begin{aligned}
\lambda= & \lambda(Z)=\lambda_{F}\left(Z_{F}\right)+\left.\frac{\mathrm{d} \lambda}{\mathrm{d} Z}\right|_{F}\left(Z-Z_{F}\right) \\
& +\left.\frac{1}{2} \frac{\mathrm{d}^{2} \lambda}{\mathrm{d} Z^{2}}\right|_{F}\left(Z-Z_{F}\right)^{2}+O\left\{\left(Z-Z_{F}\right)^{3}\right\} .
\end{aligned}
$$

Catastrophic rupture is a self-sustainable process. That is, during a catastrophic rupture, even if the controlling parameter is fixed, the accumulative response of the system varies and presents a finite jump. Thus, $\mathrm{d} Z /\left.\mathrm{d} \lambda\right|_{F}=\infty$ or $\mathrm{d} \lambda /$ $\left.\mathrm{d} Z\right|_{F}=0$ and $\mathrm{d}^{2} \lambda /\left.\mathrm{d} Z^{2}\right|_{F}<0$. With that and omitting the higher order terms in eq. (1), we obtain

$$
Z_{F}-Z \propto\left(\lambda_{F}-\lambda\right)^{\frac{1}{2}}
$$

and

$$
R_{Z}=\frac{\mathrm{d} Z(\lambda)}{\mathrm{d} \lambda} \propto\left(\lambda_{F}-\lambda\right)^{-\frac{1}{2}},\left(\text { in the vicinity of } \lambda_{F}\right)
$$

where $R_{Z}$ denotes the derivation of the accumulative response $(Z)$ of system to the controlling parameter $(\lambda)$ [18]. Eq. (3) physically means that, in the vicinity of the catastrophic rupture, the variation of the system response to the controlling parameter follows a power law with index $-1 / 2$.

In the simulation of catastrophic rupture in brittle material using the finite element method (FEM), Rong [18] found the rate of damage and energy release before catastrophe follows the power law with index $-1 / 2$. Hao studied the sample displacement in rock compression test, and found the sample displacement demonstrates power law singularity before catastrophe, and the power index varies from -0.81 to -0.30 [19].

1) PAC Corporation. PCI-2 Based AE System User's Manual, 2007

\section{Experiment}

Rectangular marble samples of dimension $50 \mathrm{~mm} \times 21.5$ $\mathrm{mm} \times 24 \mathrm{~mm}$ were uniaxially compressed with an MTS 810 material testing system. In the test, the crosshead velocity of the MTS was set as $0.05 \mathrm{~mm} / \mathrm{min}$. The displacement of the sample is measured with an extensometer whose resolution is $3 \mu \mathrm{m}$.

Generally, damage evolution in rock can been observed in the laboratory via a number of methods, such as acoustic emission (AE) [20], direct observation with Scanning Electronic Microscopy (SEM) [21] and optical microscopy [22]. In this paper, the waveform of AEs generated during microdamage propagation were detected and characterized with a Physical Acoustic Corporaton (PAC) PCI-2 based AE system. Two R15 AE sensors are fixed on two sides of the sample with a specially designed clamp. The resonant frequency of the AE sensors is approximately $150 \mathrm{kHz}$. The sampling rate is $2 \mathrm{M} / \mathrm{s}^{1)}$

\section{Results and discussion}

In Figure 1, the solid curve shows a typical nominal stressnominal strain relationship obtained in the experiment. Generally, in the initial portion of the curve, the slope $\mathrm{d} \sigma / \mathrm{d} \varepsilon$ increases due to the elastic closing of microcracks in sample. Thus, we can assume that no damage occurs during this portion of the curve and firstly we need to find the point of maximum slope, which is noted as point $A$ in Figure 1 in the curve. The elastic modulus of intact sample $E_{0}$ is defined as the slope at point $A$, that is, $E_{0}=\mathrm{d} \sigma /\left.\mathrm{d} \varepsilon\right|_{A}$. After that, a new zero point $O^{\prime}$ with intersect $\varepsilon_{0}=\varepsilon_{A}-\sigma_{A} / E_{0}$ in the strain axis is obtained, as shown in Figure 1. The secant modulus at point $B$ can be defined as:

$$
E_{B}=\frac{\sigma_{B}}{\varepsilon_{B}-\left(\varepsilon_{A}-\sigma_{A} / E_{0}\right)} .
$$

Assuming that damage in the sample results in a reduction in the secant modulus, the damage at point $B D_{B}$ can be obtained as:

$$
D_{B}=1-\frac{\sigma_{B}}{\varepsilon_{B} E_{0}-\varepsilon_{A} E_{0}+\sigma_{A}} .
$$

The dashed curve in Figure 1 shows the calculated damage versus strain.

Figure 2 shows the cumulative AE event counts and energy release versus time recorded in a typical test. Although the AE signals are generated due to the stress wave energy 


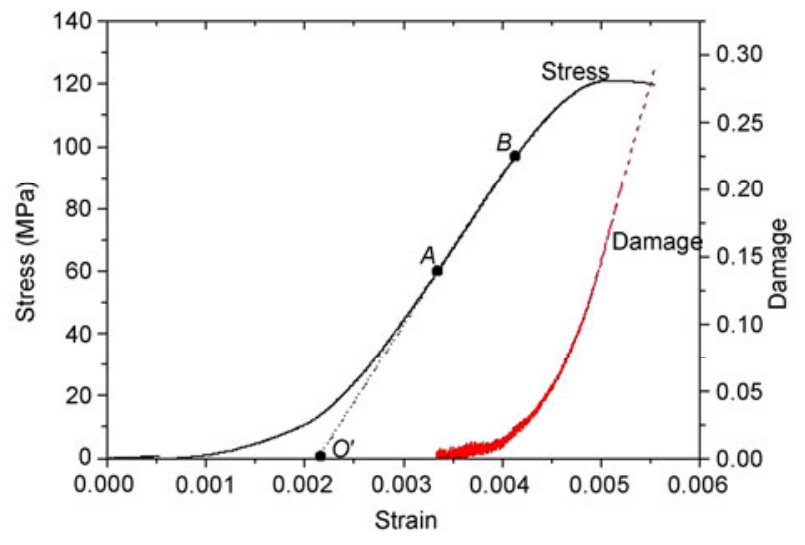

Figure 1 (Color online) Variation of stress and damage with strain. Point $A$ has the maximum slope $(\mathrm{d} \sigma / \mathrm{d} \varepsilon)$ in the stress-strain curve and the elastic modulus of intact sample $E_{0}$ is defined as $\mathrm{d} \sigma /\left.\mathrm{d} \varepsilon\right|_{A}$. The damage at point $B D_{B}$ is calculated as $D_{B}=1-\sigma_{B} /\left(\varepsilon_{B} E_{0}-\varepsilon_{A} E_{0}+\sigma_{A}\right)$.

released by the materials, the cumulative energy release is measured with a voltage-time units $(\mathrm{mV} \cdot \mu \mathrm{s})$, which is derived from the integral of the rectified voltage signal over the duration of the $\mathrm{AE}$ waveform. The rates of $\mathrm{AE}$ event counts and energy release with time are shown in Figure 3. It can be noted that just before the catastrophic point, the intervals between signals become too short to be resolved. It induces a decrease in the rate of AE event counts. This portion of data is omitted in the subsequent curve fitting.

Since AE is caused by microdamage evolution, we first compare the damage evolution with the recorded cumulative AE event counts, as shown in Figure 4. Due to sample specificity, the value of damage and cumulative AE counts varies from sample to sample. Hence, in Figure 4, both damage and the cumulative AE counts are normalized with the values at the catastrophic point of each sample. It can be seen that the data almost collapses on the straight line of the slope 1, particularly in the initial portion of the curve. That means that the cumulative AE counts are roughly proportional to the damage calculated with eq. (4). Generally speaking, AE signals reflect the microdamage events di- rectly while the macroscopic damage variable characterizes the collective effect of all the microdamages. Despite that the evolution of macroscopic damage may sensitively depend on the details of microdamage events, at early stage of damage evolution, the cumulative number of AE counts is nearly proportional to the total number of micro-damages. Based on mean field approximation, the latter is nearly proportional to the reduction in elastic modulus.

Figures 5 and 6 show the rates of AE event counts and AE energy release with time to failure before the catastrophic rupture. Similar to Figure 4, all data are normalized with the maximum value prior to the catastrophic point of each sample. Again, it can be seen that near the proximity of the catastrophic point, the curves approximately follow straight lines in a double logarithmic scale. That indicates consistency with a power law of diverse power indexes. Moreover, the negative slope of these lines also suggests that the singularity behavior of system to the controlling parameter in the vicinity of catastrophic failure. Table 1 lists the fitted power indexes for the variables and samples. It is apparent that the index varies with the variables and samples. For the rate of AE event count, the indexes vary from -1.16 to -0.77 , while for the $\mathrm{AE}$ energy release rate, the indexes vary from -1.55 to -0.91 . The data collected in $0.9 t_{F}-t_{F}$ are adopted in the curve fitting, where $t_{F}$ is the time of catastrophe rupture.

Figures 5, 6 and Table 1 demonstrate that before catastrophic rupture, the variation of the system response to the controlling parameter follows a power law of negative index. Here, the system response is $\mathrm{AE}$ counts rate and energy release rate, and the controlling parameter can be time or the crosshead displacement of the loading apparatus. The power law relationship seems to be universal for different parameters, variables and samples. Hence, power law singularity can be considered as a universal precursor of catastrophe. However, the power indexes deviate from the ideal value $-1 / 2$ obviously and present significant diversity. The uncertainty of the power indexes cannot be explained with ideal model (eq. (3)) since that the continuity assumption

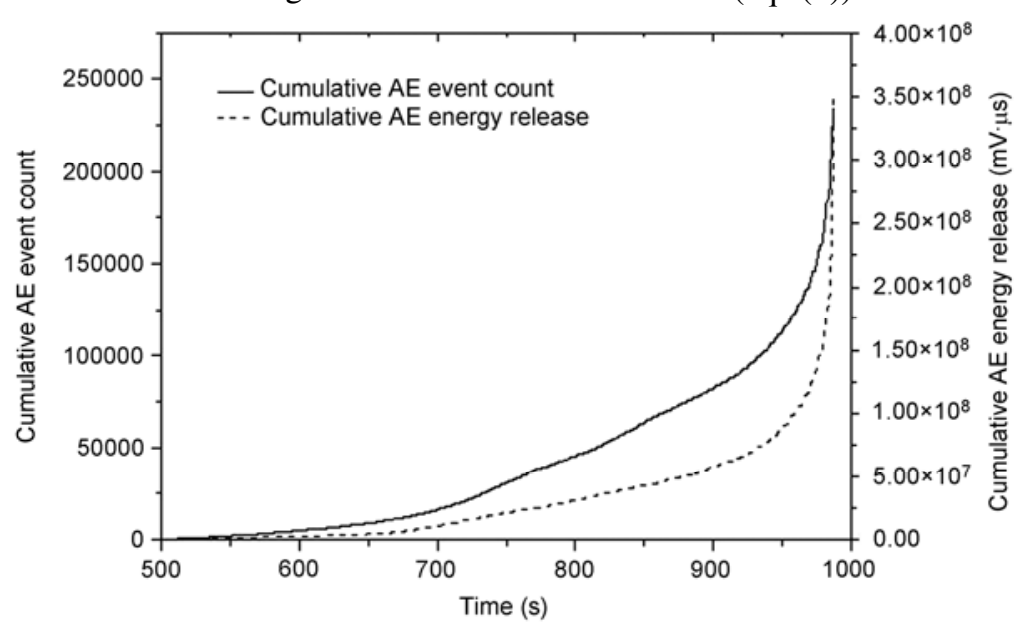

Figure 2 Variation of cumulative AE event count and cumulative AE energy release versus time recorded in a typical test. 


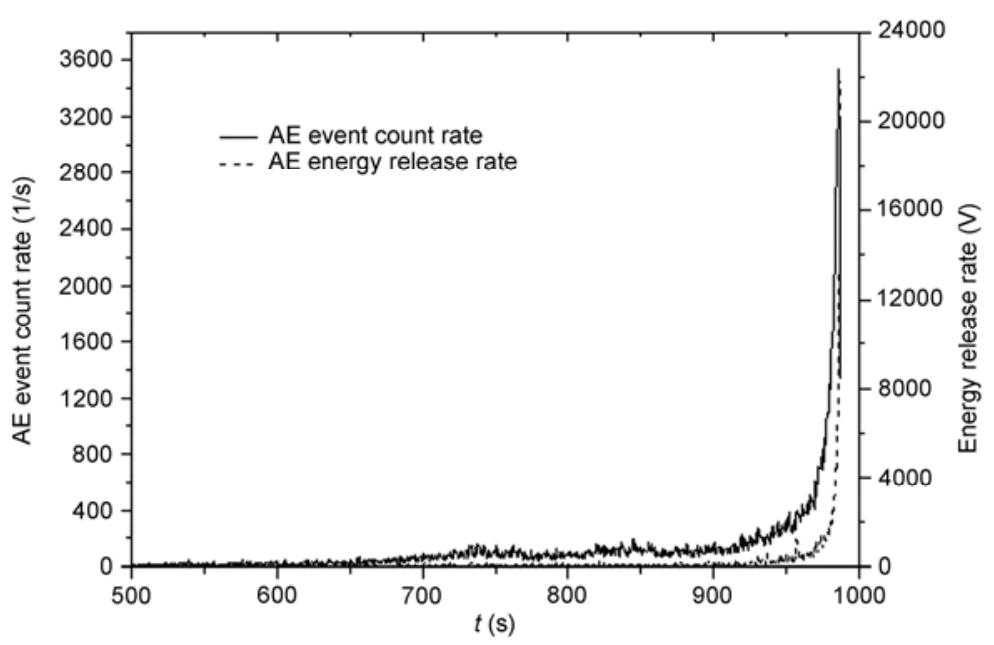

Figure 3 Variation of AE event count rate and AE energy release rate versus time in a typical test. Notice that just before the catastrophic point, the intervals between signals become too short to be resolved, which induces a decrease in the rate of AE event count.

Table 1 The fitted power indexes of AE count rate $\left(\beta_{\mathrm{N}}\right)$ and AE energy release rate $\left(\beta_{\mathrm{E}}\right)$

\begin{tabular}{cccccccccc}
\hline Sample number & 1 & 2 & 3 & 4 & 5 & 6 & 7 & 8 & 9 \\
\hline$\beta_{\mathrm{N}}$ & -0.89 & -0.98 & -0.77 & -1.14 & -0.92 & -0.92 & -1.16 & -1.12 & -1.08 \\
$\beta_{\mathrm{E}}$ & -1.43 & -1.33 & -0.91 & -1.33 & -1.46 & -1.08 & -1.55 & -1.40 & -1.26 \\
\hline
\end{tabular}

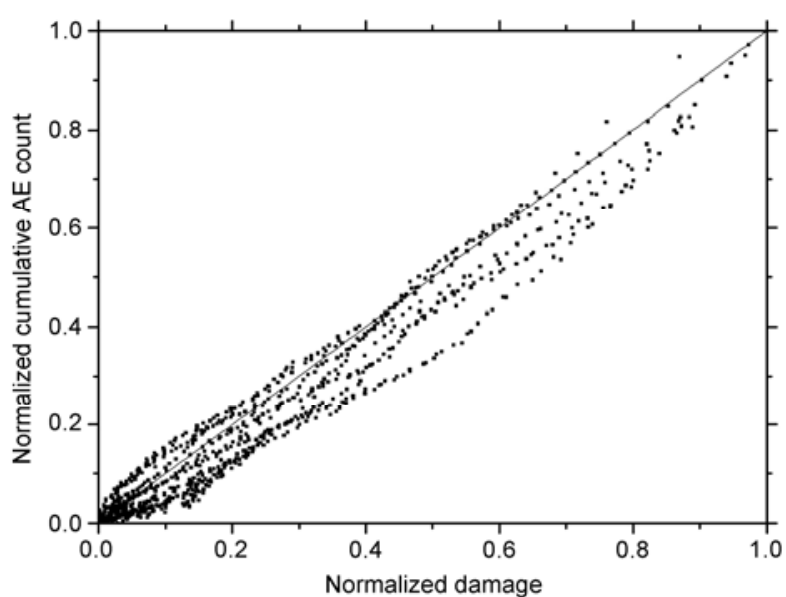

Figure 4 Variation of normalized AE count with normalized damage. The scattered points are experimental data. The slope of the solid curve is 1 .

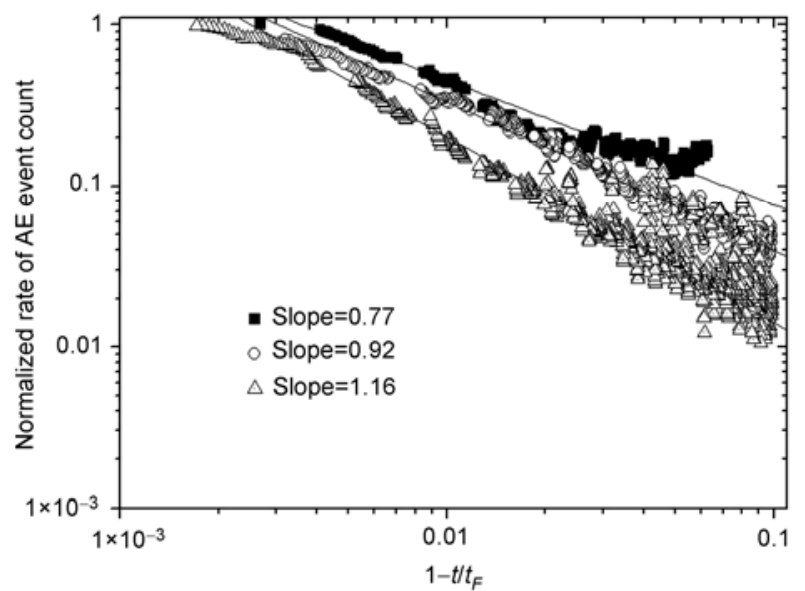

Figure 5 Variation of the normalized rate of $\mathrm{AE}$ event count with the time to failure before the catastrophic rupture. adopted in the model cannot be satisfied in our experiments. The AE signals seem to show that the damage evolution presents as a discrete and stochastic time-series. Because the size of most micro-damage events is much smaller than that of the catastrophe, the continuous and smooth model of damage evolution is approximately applicable, which results in an approximate power-law singularity. However, the randomness and the discreteness of the micro-damage events, in particular the events with larger scale, lead to the uncertainty (sample-specificity) of the power-law singularity.

\section{Conclusion}

To verify the precursor of power law singularity, we ana-

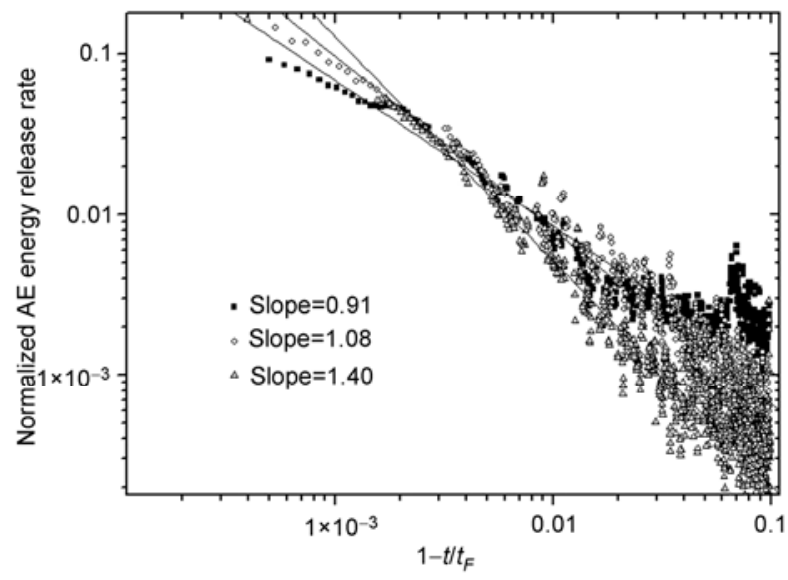

Figure 6 Variation of the normalized AE energy release rate with the time to failure before the catastrophic rupture. 
lyzed the AE signals generated by marble samples under uniaxial compression. The results show that the accumulated AE event counts is proportional to damage in the sample. In addition, prior to the catastrophic rupture, the derivative of the system response to the controlling parameter follows a power law of negative index. It is universal for different parameters, variables and samples. However, the power indexes vary with variables and samples. It reflects sample specificity and fluctuations in the evolution induced catastrophe (EIC) process. The diversity of the power index could not be explained based on the continuous model of statistical microdamage mechanics. Therefore, it is critical that the power law singularity might be a universal precursor for catastrophe prediction, but with uncertainty in the power index.

This work was supported by the National Natural Science Foundation of China (Grant Nos. 10932011, 11021262 and 11172311) and the National Basic Research Program of China (Grant No. 2012CB937500).

1 Geller R J, Jackson D D, Kagan Y Y, et al. Earthquakes cannot be predicted. Science, 1997, 275: 1616-1617

2 Wyss M. Cannot earthquakes be predicted? Science, 1997, 278: $487-490$

3 Lu M F, Li L, Xia M F, et al. Co-seismic energy release and relevant region of Tohoku M9.0 earthquake. Sci China-Earth Sci, 2011, 54(7): 947-950

4 Bai Y L, Wang H Y, Xia M F, et al. Statistical mesomechanics of solid, liking coupled multiple space and time scales. Appl Mech Rev, 2005, 58: 372-388

5 Sahimi M, Arbabi S. Mechanics of disordered solids. Phys Rev B, 1993, 47: 713-722

6 Curran D R, Seaman L, Shockey D A. Dynamic failure of solids. Phys Rep, 1987, 147: 253-388
7 Chen J K, Jiang M Q. Long-term evolution of delayed ettringite and gypsum in Portland cement mortars under sulfate erosion. Constr Build Mater, 2009, 23: 812-816

8 Bai Y L, Xia M F, Ke F J, et al. Statistical microdamage mechanics and damage field evolution. Theor Appl Fract Mech, 2001, 37: 1-10

9 Lu C S, Mai Y W, Shen Y G. Optimum information in crackling noise. Phys Rev E, 2005, 72: 027101

10 Davidsen J, Stanchits S, Dresen G. Scaling and universality in rock fracture. Phys Rev Lett, 2007, 98: 125502

11 Bhattacharyya P, Chakrabarti B K. Modelling Critical and Catastrophic Phenomena in Geoscience: A Statistical Physics Approach. Berlin Heidelberg: Springer, 2006

12 Moura A, Lei X, Nishisawa O. Self-similarity in rock cracking and related complex critical exponents. J Mech Phys Solids, 2006, 54: 2544-2553

13 Jaume S C, Sykes L R. Evolving towards a critical point: A review of accelerating seismic moment/energy release prior to large and great earthquakes. Pure Appl Geophys, 1999, 155: 279-305

14 Bowman D D, Ouillon G, Sammis C G, et al. An observation test of the critical earthquake concept. J Geophys Res, 1998, 103: 359-372

15 Rundle J B. Precursory seismic activation and critical-point phenomena. Pure Appl Geophys, 2000, 157: 2165-2182

16 Yin X C, Wang Y C, Peng K Y, et al. Development of a new approach to earthquake prediction: Load/Unload Response Ratio (LU RR) theory. Pure Appl Geophys, 2000, 157: 2365-2383

17 Xia M F, Wei Y J, Ke F J, et al. Critical sensitivity and trans-scale fluctuations in catastrophic rupture. Pure Appl Geophys, 2002, 159: 2491-2509

18 Rong F. Multiscale Numerical Simulations of Damage Evolution in Heterogeneous Brittle Media. Dissertation for Doctoral Degree. Beijing: Institute of Mechanics, Chinese Academy of Sciences, 2006

19 Hao S W. Localization, Catastrophic Rupture and Critical Singularity of Heterogeneous Brittle Media. Dissertation for Doctoral Degree. Beijing: Institute of Mechanics, Chinese Academy of Sciences, 2007

20 Lockner D A, Byerlee J D, Kuksenko V, et al. Quasi-static fault growth and shear fracture energy in granite. Nature, 1991, 350: 39-42

21 Zhao Y. Crack pattern evolution and a fractal damage constitutive model for rock. Int J Rock Mech Min Sci, 1998, 35: 349-366

22 Cox S J D, Scholz C H. Rupture initiation in shear fracture of rocks: An experimental study. J Geophys Res, 1988, 93: 3307-3320 